\title{
SUMS ARISING IN THE THEORY OF MARKOV CHAINS
}

\author{
STEVEN OREY ${ }^{1}$
}

Let $p_{\alpha \beta}^{(n)}$ be the probability of going from state $\alpha$ to state $\beta$ in $n$ steps for some recurrent irreducible Markov chain with stationary transition probabilities. It is known that for any states $i, j, k, h$ (not necessarily distinct) ${ }^{2}$

$$
\lim _{N \rightarrow \infty} \frac{\sum_{n=0}^{N} p_{i j}^{(n)}}{\sum_{n=0}^{N} p_{k h}^{(n)}}=\frac{m_{j}}{m_{h}} .
$$

Here $m_{\alpha}$ is a positive finite number, $\alpha=j, h$; in fact, $\left\{m_{\beta}\right\}$ is the invariant measure of the Markov chain. ${ }^{3}$ Here we are interested in investigating the asymptotic behavior of

$$
\sum_{n=0}^{N}\left(m_{h} p_{i j}^{(n)}-m_{j} p_{k h}^{(n)}\right)
$$

as $N \rightarrow \infty$, and also the behavior of some related sums. The sum (0.1) was first considered by Chung [1]. In special cases some results are known; we shall give detailed references to previous work in footnotes. Here we strengthen $(0.0)$ by showing that the partial sums $(0.1)$ are uniformly bounded above and below. In the ergodic aperiodic case and in certain null-recurrent cases convergence is demonstrated; these results overlap some announced in [7]. It is shown that in the null-recurrent case $(0.1)$ may fail to converge, thus settling a point raised in [1] and again in [7]; we hear $\mathrm{C}$. Stone also has given counter-examples. The behavior of $(0.1)$ and related sums has recently been shown to be of central importance for the development of a potential theory for certain recurrent Markov chains. ${ }^{4}$

We introduce some notation. Greek letters range over the state

Received by the editors October 15, 1960.

${ }_{1}$ This research was supported by the United States Air Force through the Air Force Office of Scientific Research of the Air Research and Development Command, under Contract No. AF 49(638)-617. Reproduction in whole or in part is permitted for any purpose of the United States Government.

${ }^{2}$ This ratio was considered by Doeblin. For more general results see [1].

${ }^{3}$ This is due to Derman [2].

${ }^{4}$ See Spitzer [6], Hoeffding [4], and Kemeny and Snell [7]. 
space. We use $\delta_{\alpha \beta}$ to denote 0 (1) if $\alpha \neq \beta(\alpha=\beta)$, and write " $P[\cdot]$ " for the probability of $[\cdot]$.

We define:

$$
p_{\alpha \beta}^{(n)}=P \text { [going from } \alpha \text { to } \beta \text { in } n \text { steps], } \quad n=0,1, \cdots .
$$

Note that

$$
\begin{aligned}
p_{\alpha \beta}^{(0)} & =\delta_{\alpha \beta}, \\
{ }_{\gamma} p_{\alpha \beta}^{(n)} & =P[\text { going from } \alpha \text { to } \beta \text { in } n \text { steps without visiting } \gamma \text { at any } \\
{ }_{\gamma} p_{\alpha \beta}^{(0)} & =0 .
\end{aligned}
$$

Henceforth $j$ and $k$ are two fixed distinct states. The functions we now introduce will be used only for $\alpha \in\{j, k\}, \beta \in\{j, k\}$.

$q_{\alpha \beta}^{(n)}=P$ [going from $\alpha$ to $\beta$ in $n$ steps without visiting either $j$ or $k$ $q_{\alpha \beta}^{(0)}=0$. at any positive time prior to $n], \quad n=1,2, \cdots$,

We introduce the generating functions

$$
P_{\alpha \beta}=\sum_{n=0}^{\infty} p_{\alpha \beta}^{(n)} z^{n}, \quad{ }_{\gamma} P_{\alpha \beta}=\sum_{n=0}^{\infty}{ }_{\gamma} p_{\alpha \beta}^{(n)} z^{n}, \quad Q_{\alpha \beta}=\sum_{n=0}^{\infty} q_{\alpha \beta}^{(n)} z^{n} .
$$

The first series converges in $|z|<1$, the last two in $|z| \leqq 1$. Set

$$
\pi_{\alpha \beta}=Q_{\alpha \beta}(1) \text {. }
$$

For $\alpha \in\{j, k\}, \beta \in\{j, k\}$ we have

$$
\frac{1-\pi_{\alpha \alpha}}{1-\pi_{\beta \beta}}=\frac{m_{\beta}}{m_{\alpha}} .
$$

This follows easily from the identities of $\$ 1$ and Derman's relationship

$$
{ }_{\alpha} P_{\alpha \beta}(1)=\frac{m_{\beta}}{m_{\alpha}} .
$$

Since the invariant measure is determined only up to a constant multiple we may and shall assume

$$
\left(1-\pi_{k k}\right)=m_{j}, \quad\left(1-\pi_{j j}\right)=m_{k} .
$$

We shall be concerned with the functions 


$$
\begin{aligned}
& S_{\alpha \beta}=m_{\alpha}\left(P_{\beta \beta}-P_{\alpha \beta}\right), \\
& T_{\alpha \beta}=m_{\beta} P_{\alpha \alpha}-m_{\alpha} P_{\alpha \beta} .
\end{aligned}
$$

We write

$$
\begin{aligned}
& S=S_{j k}=\left(1-\pi_{k k}\right)\left(P_{k k}-P_{j k}\right), \\
& T=T_{j k}=\left(1-\pi_{j j}\right) P_{j j}-\left(1-\pi_{k k}\right) P_{j k} .
\end{aligned}
$$

If $E$ is an expression depending on $j$ and $k$ we write $E^{*}$ for the expression obtained by interchanging the roles of $j$ and $k$. Thus $S^{*}=S_{k j}$, $T^{*}=T_{k j}$.

1. Basic identities. ${ }^{5}$ The basic relation of renewal theory is

$$
P_{j j}=\frac{1}{1-{ }_{j} P_{j j}} .
$$

This identity, and all others we introduce, will hold for $|z|<1$.

By an obvious argument one obtains

$$
P_{j k}=P_{j j}, P_{j k}={ }_{k} P_{j k} P_{k k} .
$$

Similarly one obtains

$$
\begin{aligned}
{ }_{k} P_{j k} & =\frac{Q_{j k}}{1-Q_{j j}}, \\
{ }_{j} P_{j j} & =Q_{j j}+Q_{j k} P_{k j}=Q_{j j}+\frac{Q_{j k} Q_{k j}}{1-Q_{k k}}, \\
{ }_{k} P_{j j} & =\frac{Q_{j j}}{1-Q_{j j}}, \\
{ }_{j} P_{j k} & =Q_{j k}\left(1+{ }_{j} P_{k k}\right)=\frac{Q_{j k}}{1-Q_{k k}} .
\end{aligned}
$$

The formulas obtained by interchanging $j$ and $k$ will be called conjugate to the original formulas.

It is important to note that the $Q_{\alpha \beta}$ are arbitrary in the following precise sense: given any constants $q_{\alpha \beta}^{(n)}, n=1,2, \cdots, \alpha \in\{j, k\}$, $\beta \in\{j, k\}$, there exists a recurrent irreducible Markov chain with states $j$ and $k$ (and possibly other states) such that

$$
Q_{\alpha \beta}=\sum_{n=0}^{\infty} q_{\alpha \beta}^{(n)} z^{n}
$$

SIdentity (1.2) is due to Chung; it was his idea to exploit relationships involving one "taboo" state. (1.3)-(1.6) involve a double taboo. 
provided only the following conditions hold:

$$
\begin{gathered}
0 \leqq q_{\alpha \beta}^{(n)}, \quad n=1,2, \cdots, \alpha \in\{j, k\}, \beta \in\{j, k\} . \\
\sum_{m=1}^{\infty}\left(q_{j k}^{(m)}+q_{j j}^{(m)}\right)=\sum_{m=1}^{\infty}\left(q_{k j}^{(m)}+q_{k k}^{(m)}\right)=1, \\
\left(\sum_{m=1}^{\infty} q_{j k}^{(m)}\right)\left(\sum_{m=1}^{\infty} q_{k j}^{(m)}\right)>0 .
\end{gathered}
$$

2. Summability and boundedness. We introduce the following quantities:

$$
\begin{aligned}
& A=1-Q_{j j}-Q_{j k}, \\
& B=1-Q_{k k}+Q_{k j}, \\
& C=Q_{k j}\left(1-Q_{j j}\right)-Q_{j k}\left(1-Q_{k k}\right), \\
& D=\left(1-Q_{j j}\right)\left(1-Q_{k k}\right)-Q_{j k} Q_{k j} .
\end{aligned}
$$

These quantities are defined in $|z| \leqq 1$.

Recalling the definition of $S$ given by $(0.4),(0.6)$ and substituting the identites of $\$ 1$ (or the conjugates) one obtains

$$
S=\frac{\left(1-\pi_{k k}\right) A}{D}
$$

Similarly $T$, which is defined by $(0.5)$ and (0.6) turns out to satisfy

$$
T=\frac{\left(1-\pi_{j j}\right)-\left[\left(1-\pi_{j j}\right) Q_{k k}+\left(1-\pi_{k k}\right) Q_{j k}\right]}{D} .
$$

The following identity, which is an immediate consequence of the definition of $D$ and the identities in $\$ 1$ and their conjugates, will be used frequently.

$$
D=\left(1-{ }_{j} P_{j j}\right)\left(1-Q_{k k}\right)=\left(1-{ }_{k} P_{k k}\right)\left(1-Q_{j j}\right) .
$$

LEMMA. Let

$$
S=\sum_{n=0}^{\infty} s_{n} z^{n}, \quad T=\sum_{n=0}^{\infty} t_{n} z^{n}
$$

Then

$$
0<\sum_{n=0}^{N} s_{n}<1, \quad \text { and } \quad 0<\sum_{n=0}^{N} t_{n}, \quad N=0,1, \cdots
$$

Proof. From (2.1) and (2.3) we obtain 


$$
S=\frac{A}{1-{ }_{j} P_{j j}} \cdot \frac{1-\pi_{k k}}{1-Q_{k k}} .
$$

The second factor gives rise to a power series with non-negative coefficients and converging to 1 when $z=1$. Let

$$
\frac{A}{1-{ }_{j} P_{j j}}=\sum_{n=0}^{\infty} h_{n} z^{n}, \quad A=\sum_{n=0}^{\infty} a_{n} z^{n} .
$$

Remembering (1.1) one finds that

$$
\sum_{n=0}^{N} h_{n}=\sum_{v=0}^{N} p_{j j}^{(v)} \sum_{m=0}^{N-v} a_{m}=\sum_{v=0}^{N} p_{j j}^{(v)} \sum_{m=N=v+1}^{\infty}\left(q_{j j}^{(m)}+q_{j k}^{(m)}\right) .
$$

From (1.1) one easily derives

$$
\sum_{v=0}^{N} p_{j j}^{(v)} \sum_{m=N-v+1}^{\infty}{ }_{j=0}^{(m)}=1, \quad N=0,1,2, \cdots .
$$

Notice that

$P$ [first return to $j$ occurs after time $N-v \mid$ start at $j]$

$$
\begin{aligned}
& =\sum_{m=N-v+1}^{\infty} p_{j j}^{(m)} \\
& \geqq P[\text { first return to set }\{j, k\} \text { occurs after time } N-v \mid \text { start at } j] \\
& =\sum_{m=N-v+1}^{\infty}\left(q_{j j}^{(m)}+q_{j k}^{(m)}\right) .
\end{aligned}
$$

Clearly the first part of our lemma now follows. The assertion about $T$ is proved quite similarly.

Theorem $1 .^{6}$ For any states $\alpha, \beta, \gamma, \delta$, not necessarily distinct, there exists a finite positive $M$ such that

$$
\left|\sum_{n=0}^{N}\left[m_{\delta} p_{\alpha \beta}^{(n)}-m_{\beta} p_{\gamma \delta}^{(n)}\right]\right|<M, \quad N=0,1, \cdots .
$$

Proof. From the previous lemma it is clear that for any two states $\alpha, \beta S_{\alpha \beta}(1)$ and $T_{\alpha \beta}(1)$ each represent a series all of whose partial sums are positive, and such that the partial sums of $S_{\alpha \beta}(1)$ are bounded uniformly from above (however the bound 1 obtained in the lemma for $\alpha=j, \beta=k$ is due to the normalization (0.3)). The theorem then follows from ding.

- The special case $\alpha=\beta=\gamma$ of this theorem has been proved earlier by W. Hoeff- 


$$
\begin{aligned}
m_{\delta} & P_{\alpha \beta}-m_{\beta} P_{\gamma \delta} \\
& =S_{\gamma \delta}\left(m_{\beta} / m_{\gamma}\right)-T_{\delta \alpha}\left(m_{\beta} / m_{\alpha}\right)+S_{\delta \alpha}\left(m_{\beta} / m_{\alpha}\right)-T_{\alpha \beta}\left(m_{\delta} / m_{\alpha}\right) \\
& =-S_{\alpha \beta}\left(m_{\delta} / m_{\alpha}\right)+T_{\beta \gamma}\left(m_{\delta} / m_{\gamma}\right)-S_{\beta \gamma}\left(m_{\delta} / m_{\gamma}\right)+T_{\gamma \delta}\left(m_{\beta} / m_{\gamma}\right) .
\end{aligned}
$$

Note that $\alpha=\beta$ implies $S_{\alpha \beta}=T_{\alpha \beta}=0$.

We shall now consider $S+S^{*}$. Observe that

$$
\begin{aligned}
& D=A B-C=A^{*} B^{*}-C^{*}=D^{*}, \\
& C=-C^{*} .
\end{aligned}
$$

Adding (2.1) to its conjugate and using (2.9) we obtain after simplifying

$$
\begin{aligned}
S+S^{*}= & \frac{1-\pi_{k k}}{B}+\frac{1-\pi_{j j}}{B^{*}} \\
& +\frac{1}{B B^{*}}\left[\frac{\left(1-\pi_{k k}\right) B^{*} C+C^{*} B\left(1-\pi_{j j}\right)}{D}\right] .
\end{aligned}
$$

We shall write

$$
Q_{\alpha \beta}=\pi_{\alpha \beta}-\epsilon_{\alpha \beta} .
$$

Note that $\epsilon_{\alpha \beta}$ is continuous in $|z| \leqq 1, \epsilon_{\alpha \beta}(1)=0$, and $\epsilon_{\alpha \beta}(z)>0$ for $0<z<1$.

The following theorem now is easy.

TheOREM $2 .{ }^{7} S+S^{*} \rightarrow 1$ as $z \rightarrow 1-$.

Proof. Inspection of (2.11) reveals that we need simply show that

$$
\lim _{z \rightarrow 1-} \frac{\left(1-\pi_{k k}\right) B^{*} C+C^{*} B\left(1-\pi_{j j}\right)}{D}=0 .
$$

We call a term of the form $\epsilon_{\alpha \beta}$ an $\epsilon$-term, and a product of two or more such terms a higher-order $\epsilon$-term. One finds that

$$
\begin{aligned}
D= & \left(1-\pi_{j j}\right)\left[\epsilon_{k k}+\epsilon_{k j}\right]+\left(1-\pi_{k k}\right)\left[\epsilon_{j j}+\epsilon_{j k}\right] \\
& + \text { (higher order } \epsilon \text {-terms). }
\end{aligned}
$$

7 In other words we establish Abel summability of a certain series. In [5] it was shown that for the special case considered there this series actually converges to 1 . Since convergence was easier to prove than that the sum is 1 the present theorem leads to a shorter proof of that result of [5].

W. Hoeffding has communicated to us that in the aperiodic case he can establish convergence to one of the series in question, by using a recent result of Chung. 
On the other hand

(2.15) $\left(1-\pi_{k k}\right) B^{*} C+C^{*} B\left(1-\pi_{j j}\right)=0+$ (higher order $\epsilon$-terms).

Keeping in mind the remarks after (2.12) one sees that the theorem is established. We note that $T+T^{*}=S+S^{*}$.

TheOREM $3 .^{8}$ As $z \rightarrow 1-, S$ approaches a limit if and only if $\left(\epsilon_{j j}+\epsilon_{j k}\right) /\left(\epsilon_{k k}+\epsilon_{k j}\right)$ approaches a limit as $z \rightarrow 1-$.

Proof. Note that $A=\epsilon_{j j}+\epsilon_{j k}$. Formulas (2.1), (2.14), and the remarks after (2.12) show the truth of the theorem.

3. Convergence and absolute convergence. If

$$
H=\sum_{n=0}^{\infty} h_{n} z^{n}, \quad|z|<1,
$$

we shall write

$$
\begin{array}{ll}
H \in \mathcal{C} & \text { for } \sum_{n=0}^{\infty} h_{n} \text { converges, } \\
H \in Q & \text { for } \sum_{n=0}^{\infty}\left|h_{n}\right| \text { converges, } \\
H \in Q & \text { for } B \in \mathcal{C} \text { and } \frac{H(1)-H(z)}{1-z} \in Q .
\end{array}
$$

Note that

$$
\sum_{n=0}^{\infty} n\left|h_{n}\right|<\infty
$$

implies $H \in R$.

The Markov chain is said to be periodic if there exists an integer $w$ greater than 1 such that

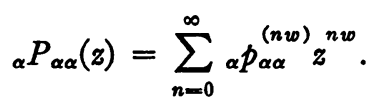

Because of the assumption of irreducibility this definition is independent of $\alpha$. If the chain is not periodic it is aperiodic. The chain is positive recurrent if ${ }_{\alpha} P_{\alpha \alpha} \in R$; this definition too is independent of $\alpha$. A recurrent chain that is not positive recurrent will be called null recurrent.

8 Remember that the $\epsilon_{\alpha \beta}$ are arbitrary. So one can produce examples in which the series $S$ considered at $z=1$ does not Abel converge; a fortiori it will not converge. 
THEOREM $4 .^{9}$ In the positive recurrent, aperiodic case

$$
\left[m_{\delta} P_{\alpha \beta}-m_{\beta} P_{\gamma \delta}\right] \in a, \quad \text { for all states } \alpha, \beta, \gamma, \delta .
$$

Proof. In view of (2.8) it is only necessary to show that under the hypotheses of the theorem we have $S \in Q$ and $T \in Q$. The proof is similar for $S$ and $T$; we give it for $T$. Using (2.2) and (2.3) we obtain

$$
T=\frac{\left(1-\pi_{j j}\right)-\left[\left(1-\pi_{j j}\right) Q_{k k}+\left(1-\pi_{k k}\right) Q_{j k}\right]}{1-{ }_{k} P_{k k}} \cdot \frac{1}{1-Q_{j j}}
$$

By the assumption of the theorem ${ }_{k} P_{k k} \in R$, that is the expected recurrence time for the state $k$ is finite. It is evident that then $Q_{k k} \in R$ and $Q_{j k} \in R$; this can easily be proved by using an argument similar to that expressed by (2.7). Rewriting the right hand side of (3.1) we have

$$
\begin{gathered}
T=\left\{\left(1-\pi_{j j}\right)\left[\frac{\pi_{k k}-Q_{k k}}{1-z}\right]+\left(1-\pi_{k k}\right)\left[\frac{\left(1-\pi_{j j}\right)-Q_{j k}}{1-z}\right]\right\} \\
\cdot \frac{1-z}{1-{ }_{k} P_{k k}} \cdot \frac{1}{1-Q_{j j}} .
\end{gathered}
$$

Since $Q_{k k} \in R$ and $Q_{j k} \in R$ the first factor on the right of (3.2) belongs to $a$. That $(1-z) /\left(1-{ }_{k} P_{k k}\right) \in Q$ when the hypotheses of the theorem hold follows from Wiener's tauberian theorem, ${ }^{10}$ and clearly $\left[1-Q_{j j}\right]^{-1}$ $\in Q$. Thus the theorem is established.

It is easy to obtain an explicit expression for the quantity $\left[m_{\delta} P_{\alpha \beta}-m_{\beta} P_{\gamma \delta}\right](1)$. In case $\beta=\delta$ this quantity can be expressed in terms of the moments

$$
\sum_{n=0}^{\infty} n_{\beta} p_{\alpha \beta}^{(n)}, \quad \alpha \in\{a, b, c, d\}, \quad \beta \in\{a, b, c, d\}
$$

and thus the formula in Theorem 5 of Chung [1] can be obtained;

${ }^{9}$ This theorem should be compared with Theorem 5 of [1]. In the periodic case Chung's theorem must be modified, for a two state chain in which the states alternate with probability one provides a counterexample. Chung has presented his theorem with a new proof as Theorem 4 on p. 62 of his book Markov chains with stationary transition probabilities, Berlin, 1960. He communicates the following correction for the periodic case: in case the process has period $d>1$ the series may fail to converge, however the limit as $N \rightarrow \infty$ of the $(N d+r)$ th partial sum converges to the given value, for $0 \leqq r<d$. The proof needs no change, except for a trivial modification in the last displayed formula.

${ }^{10}$ See $[3]$ for details. 
(note that his sum starts with $n=1$, ours with $n=0$ ). However, when $\beta \neq \delta$ the desired quantity cannot be expressed in terms of the moments (3.3).

If the assumption of aperiodicity in Theorem 3.4 is dropped, the theorem becomes false, even if the conclusion is weakened by replacing $a$ by $\mathfrak{e}$; see footnote 9 .

We turn to the questions of convergence and absolute convergence in the null recurrent case.

Wiener's tauberian theorem tells us that $B^{-1} \in Q$ and $\left(B^{*}\right)^{-1} \in Q$ provided only that

$$
B \cdot B^{*}=0 \text { has no roots in }|z| \leqq 1 \text {. }
$$

Clearly any roots of $B \cdot B^{*}=0$ in $|z| \leqq 1$ must lie on $|z|=1$. Note that $B=0$ on $|z|=1$ implies that there exists an integer $w$ such that $Q_{k k}$ is a power series in even powers of $z^{w}$ and $Q_{j k}$ is a power series in odd powers of $z^{w}$; and dually for $B^{*}$.

THEOREM 5. In the null recurrent case we have

(i) $\left[\left(1-\pi_{j j}\right)\left(1-Q_{k k}\right)-\left(1-\pi_{k k}\right) Q_{j k}\right]-\left[\left(1-\pi_{k k}\right)\left(1-Q_{j j}\right)-\left(1-\pi_{j j}\right) Q_{k j}\right]$ $\in R$ implies $T-T^{*} \in \mathcal{C}$ and $\left(T-T^{*}\right)(1)=0$.

(ii) $\left[\left(1-\pi_{k k}\right) A-\left(1-\pi_{j j}\right) A^{*}\right] \in \mathbb{R}$ implies $S-S^{*} \in \mathfrak{C}$ and $\left(S-S^{*}\right)(1)=0$.

(iii) If neither $B=0$ or $B^{*}=0$ has roots in $|z| \leqq 1$ and $\left[\left(1-\pi_{k k}\right) B^{*} C+C^{*} B\left(1-\pi_{j j}\right)\right] \in \mathbb{R}$ then $T+T^{*}=S+S^{*} \in \mathfrak{C}$ and $\left(S+S^{*}\right)(1)=1$.

Proof. The proof of (i) is like that of (ii) so we shall omit it.

Using (2.1) and its conjugate and (2.3) one obtains

$$
S-S^{*}=\frac{\left(1-\pi_{k k}\right) A-\left(1-\pi_{j j}\right) A^{*}}{1-z} \cdot \frac{1-z}{1-{ }_{j} P_{j j}} \cdot \frac{1}{1-Q_{k k}}
$$

That the first factor on the right of (3.7) belongs to $a$ easily follows from the hypothesis of (ii); the last factor clearly belongs to $a$. The second factor gives rise to a power series which converges to 0 when $z=1$; this is the content of the renewal theorem in the null-recurrent case. ${ }^{11}$ Now (ii) follows.

Now assume the hypotheses of (iii). The assumption on $B$ and $B^{*}$ allows us to apply Wiener's tauberian theorem to conclude that the first two terms on the right of (2.11) belong to $a$. That the last term on the right of (2.11) belongs to $\mathfrak{C}$ is proved by the same argument used to establish (ii). That $\left(S+S^{*}\right)(1)=1$ follows from Theorem 2 .

${ }^{11}$ See [3]. 
CoROllaRy 1. If the hypotheses of (i), (ii), and (iii) are satisfied $S \in \mathfrak{C}, T \in \mathcal{C}$ and $S(1)=1 / 2, T(1)=1 / 2$.

The hypothesis of the last corollary imposes conditions on $j$ and $k$. Let us say that $\left({ }^{*}\right)$ holds if the corresponding conditions are satisfied by every pair of distinct states. If the state space is a vector space and $P_{\alpha \beta}=g(\alpha-\beta)=g(\beta-\alpha)$ and the chain is aperiodic $\left(^{*}\right)$ will hold.

Corollary 2. If $\left({ }^{*}\right)$ holds $\left[m_{\delta} P_{\alpha \beta}-m_{\beta} P_{\gamma \delta}\right] \in \mathcal{C}$ for all states $\alpha, \beta$, $\boldsymbol{\gamma}, \boldsymbol{\delta}$.

Proof. Assume $\left({ }^{*}\right)$; the desired conclusion follows at once from Corollary 1 and (2.8). It is easy to write an explicit expression for the value of the sum.

\section{BIBLIOGRAPHY}

1. K. L. Chung, Contributions to the theory of Markov chains, J. Res. Nat. Bur. Standards vol. 50 (1953) pp. 203-208.

2. C. Derman, $A$ solution to a set of fundamental equations in Markov chains, Proc. Amer. Math. Soc. vol. 5 (1954) pp. 332-334.

3. P. Erdös, W. Feller, and H. Pollard, $A$ property of power series with positive coefficients, Bull. Amer. Math. Soc. vol. 55 (1949) pp. 201-204.

4. W. Hoeffding, On sequences of sums of independent random vectors, 1960 Berkeley Symposium for Probability and Statistics, to appear.

5. F. Spitzer, Some properties of recurrent random walk, Illinois J. Math, to appear.

6. - Recurrent random walk and logarithmic potential, 1960 Berkeley Symposium for Probability and Statistics, to appear.

7. J. G. Kemeny and J. L. Snell, Potentials for denumerable Markov chains, (Abstract), Dartmouth Mathematics Project, Progress Report no. 6, 1960.

UNIVERSITY OF MINNESOTA 